\title{
Micromechanical study of elastic moduli of loose granular materials
}

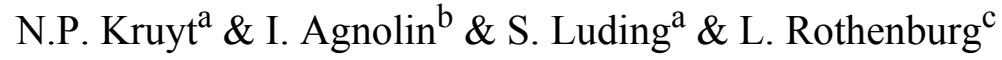 \\ ${ }^{a}$ Department of Mechanical Engineering, University of Twente, Enschede, The Netherlands \\ ${ }^{b}$ Deformation and Rheology Department, GeoForschungsZentrum Potsdam, Potsdam, Germany \\ ${ }^{c}$ Department of Civil Engineering, University of Waterloo, Waterloo, Canada \\ Journal of the Mechanics and Physics of Solids \\ (accepted for publication)
}

\begin{abstract}
In micromechanics of the elastic behaviour of granular materials, the macro-scale continuum elastic moduli are expressed in terms of micro-scale parameters, such as coordination number (the average number of contacts per particle) and interparticle contact stiffnesses in normal and tangential directions. It is well-known that mean-field theory gives inaccurate micromechanical predictions of the elastic moduli, especially for loose systems with low coordination number.

Improved predictions of the moduli are obtained here for loose two-dimensional, isotropic assemblies. This is achieved by determining approximate displacement and rotation fields from the force and moment equilibrium conditions for small sub-assemblies of various sizes. It is assumed that the outer particles of these sub-assemblies move according to the mean field. From the particle displacement and rotation fields thus obtained, approximate elastic moduli are determined. The resulting predictions are compared with the true moduli, as determined from Discrete Element Method simulations for low coordination numbers and for various values of the tangential stiffness (at fixed value of the normal stiffness). Using this approach, accurate predictions of the moduli are obtained, especially when larger sub-assemblies are considered.

As a step towards an analytical formulation of the present approach, it is investigated whether it is possible to replace the local contact stiffness matrices by a suitable average stiffness matrix. It is found that this generally leads to a deterioration of the accuracy of the predictions.

Many micromechanical studies predict that the macroscopic bulk modulus is hardly influenced by the value of the tangential stiffness. It is shown here from Discrete Element Method simulations of hydrostatic compression that for loose systems, the bulk modulus strongly depends on the stiffness ratio for small stiffness ratios.
\end{abstract}




\section{Introduction}

Knowledge of the mechanical behaviour of granular materials is important in many industrial, geotechnical and geophysical applications. This behaviour is described by the constitutive relation, which usually is based on a continuum-mechanical approach, and involves heuristic assumptions.

An alternative is the micromechanical approach (or multi-scale approach), in which a granular material is modelled as an assembly of interacting particles. The challenge is then to predict the macro-level, continuum behaviour in terms of micro-level characteristics of particles and interparticle contacts. The micromechanical approach therefore incorporates the discrete nature of granular materials.

Two special features of granular materials that make their behaviour different from many other materials are that (i) the particles have rotational degrees of freedom, besides the standard translational degrees of freedom; thus extended continuum mechanical theories are appropriate (see for example Eringen, 1999) and (ii) the particle interaction is non-central. This can be modelled by tangential elasticity and/or Coulomb friction at interparticle contacts.

Here micromechanics of quasi-static deformation of granular materials is studied. The continuum constitutive relation then involves (increments of) the stress and strain tensors. The focus is on the elastic behaviour of two-dimensional isotropic systems at low packing densities. True, elastic and reversible deformation of cohesionless granular materials is found for very small strains, typically for strains of $10^{-3} \%$ for stress levels encountered in engineering applications (Tatsuoka, 1999) or in dynamic problems. For larger strains, true reversible elastic behaviour is lost, due to frictional behaviour and due to disruption and creation of contacts between particles (see for example, Calvetti et al., 2002; Rothenburg \& Kruyt, 2004).

An overview of experimental results and methods on the elastic behaviour of granular materials is presented by Tatsuoka (1999). Measurements of elastic moduli, using static methods, are presented by Hoque \& Tatsuoka (2004). Magnanimo et al. (2008) list various studies wherein the elastic moduli have been determined from dynamic, wave-speed measurements.

In many micromechanical studies of the elastic moduli (Rothenburg, 1980; Christoffersen et al., 1981; Digby, 1981; Walton, 1987; Bathurst and Rothenburg, 1988a, b; Chang et al., 1990; Rothenburg and Bathurst, 1992; Chang and Liao, 1994; Cambou et al., 1995; Luding, 2004), the uniform strain assumption (or mean field assumption) is adopted, according to which the relative displacement of two particles in contact is determined by the average displacement gradient and the relative position vector of the particle centres. However, the corresponding predictions of the moduli generally significantly overestimate the actual moduli, especially for loose systems for which the coordi- 


\section{N.P. Kruyt \& I. Agnolin \& S. Luding \& L. Rothenburg}

nation number (the average number of contacts per particle) of the assembly is low (see for example Kruyt \& Rothenburg, 1998; Makse et al., 1999; Rothenburg \& Kruyt, 2001; Agnolin \& Roux, 2007b; Somfai et al., 2007; Magnanimo et al., 2008). Using minimum potential energy principles, it can be shown rigorously that the uniform strain assumption gives an upper bound to the moduli (Kruyt \& Rothenburg, 2002, 2004). Another lower bound for the elastic bulk modulus is given by Agnolin \& Roux (2007b).

As noted before, the relative displacements of particles in contact deviate from that according to the uniform strain assumption. These deviations, or fluctuations, have been studied systematically from results of Discrete Element Method (DEM for short) simulations (Kruyt \& Rothenburg, 1998; Agnolin \& Roux, 2007b; Agnolin \& Kruyt, 2008). Theoretical work has been done to predict these fluctuations and, as a result, obtain more accurate predictions of the elastic moduli. In most studies (except Rothenburg \& Kruyt, 2009) the fluctuations are determined by solving the displacements and rotations from the equilibrium equations for very small sub-assemblies. This work is either purely analytical (Jenkins et al., 2005; Agnolin et al., 2006; La Ragione \& Jenkins, 2007) or seminumerical (Kruyt \& Rothenburg, 2002, 2004; Agnolin \& Roux, 2008; Agnolin \& Kruyt, 2008).

Such approaches work reasonably well for dense assemblies with high coordination number, while for loose assemblies with low coordination number large deviations are observed between results of DEM simulations and theoretical predictions (Kruyt \& Rothenburg, 2002; Agnolin \& Roux, 2007b, 2008; Agnolin \& Kruyt, 2008). The focus of the current study is therefore on the 'difficult' case of loose assemblies with low coordination number. Previous approaches are extended here by solving displacements and rotations fluctuations from the equilibrium conditions for subassemblies with various sizes.

The outline of this paper is as follows. In Section 2 an overview is given of micromechanics of granular materials. In Section 3 the adopted approach is described for the determination of the particle displacement and rotation field from equilibrium conditions for small sub-assemblies of particles. From these fields, predictions of the moduli are obtained. In Section 4 the DEM simulations, as proposed by Cundall \& Strack (1979), are described. In Section 5 the predictions from the micromechanical approach are compared against those from the DEM simulations.

The usual sign convention from continuum mechanics is employed for stress and strain, according to which tensile stresses and strains are considered positive. The summation convention is adopted, implying a summation over repeated subscripts. 


\section{Micromechanics}

In the absence of long-range forces, particles only exert forces on one another when they are in contact. The particles in the assembly are assumed to be semi-rigid, so that the interparticle interaction can be considered as point forces acting at contacts.

Here assemblies of two-dimensional circular particles, i.e. disks, are considered. The radius of particle $p$ is denoted by $R^{p}$. The geometry of two particles $p$ and $q$ in contact is depicted in Figure 1 for the two-dimensional case: $n_{i}^{p q}$ and $t_{i}^{p q}$ are the normal and tangential vector at the contact point $c$, respectively. The position vector of the centre of particle $p$ is denoted by $X_{i}^{p}$. The branch vector $l_{i}^{p q}$ is the vector connecting the centres of two particles in contact, i.e. $l_{i}^{p q}=X_{i}^{q}-X_{i}^{p}$. The orientation of a contact $\theta^{p q}$ is defined by the direction of its branch vector $n_{i}^{p q}=\left(\cos \theta^{p q}, \sin \theta^{p q}\right)^{T}($ see also Figure 1).

Statistical properties of the contact geometry of an assembly are primarily described by the two quantities: (i) the coordination number $\Gamma$, i.e. the average number of contacts per particle, which is a measure of the packing density (Quickenden \& Tan, 1974; Kruyt \& Rothenburg, 1996) and (ii) the contact distribution function $E(\theta)$ (Horne, 1965) that is defined such that $E(\theta) \Delta \theta$ gives the fraction of contacts with orientation $\theta$ within the interval $(\theta, \theta+\Delta \theta)$. For the two-dimensional isotropic assemblies considered here, $E(\theta)=1 /(2 \pi)$. Note that there is no one-to-one relation between packing density and coordination number (Agnolin \& Roux, 2007a; Magnanimo et al., 2008).

In the absence of body forces, the quasi-static two-dimensional force and moment equilibrium conditions for particle $p$ are

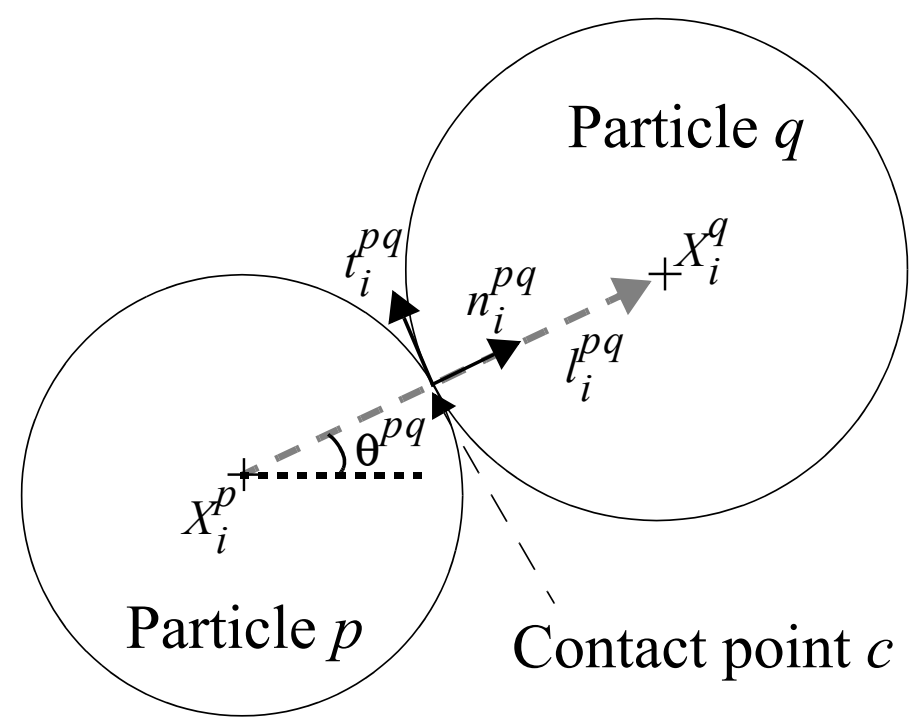

Figure 1. Contact geometry of particles $p$ and $q$, involving the contact normal and tangential vectors $n_{i}^{p q}$ and $t_{i}^{p q}$, branch vector $l_{i}^{p q}$ and contact orientation $\theta^{p q}$. 


$$
\sum_{q} f_{i}^{p q}=0 \quad e_{i j} \sum_{q} r_{i}^{p q} f_{j}^{p q}=0
$$

where $r_{i}^{p q}$ is the vector from the centre of particle $p$ to the point of contact between particles $p$ and $q, f_{i}^{p q}$ is the force exerted on particle $p$ by particle $q$, the summation is over the particles $q$ that are in contact with particle $p$ and $e_{i j}$ is the two-dimensional permutation symbol.

Using an averaging technique for the force equilibrium conditions Eq. (1), the micromechanical expression for the average (increment of the) Cauchy stress tensor $\sigma_{i j}$ is obtained (see for example, Drescher \& de Josselin de Jong, 1972; Kruyt \& Rothenburg, 1996)

$$
\sigma_{i j}=\frac{1}{A} \sum_{c \in C} f_{i}^{c} l_{j}^{c}
$$

where $A$ is the total area occupied by the assembly (including voids) and $C$ is the set of contacts. Note that $f_{i}^{c} l_{j}^{c}$ is a 'proper' contact quantity: $f_{i}^{q p} l_{j}^{q p}=f_{i}^{p q} l_{j}^{p q}$, since $l_{j}^{q p}=-l_{j}^{p q}$ and $f_{i}^{q p}=-f_{i}^{p q}$. The latter identity is Newton's third law.

The relative displacement $\Delta_{i}^{p q}$ at the contact between particles $p$ and $q$ consists of two terms, one due to the displacement of the particle centres and one due to the rotation of particles

$$
\Delta_{i}^{p q}=\left[U_{i}^{q}+\Omega^{q} e_{j i} r_{j}^{q p}\right]-\left[U_{i}^{p}+\Omega^{p} e_{j i} r_{j}^{p q}\right]
$$

where $U_{i}^{p}$ is the displacement of the centre of particle $p$ and $\Omega^{p}$ is its rotation (counted positive in counter-clockwise direction). Note that $\Delta_{i}^{q p}=-\Delta_{i}^{p q}$.

The link between (increments of) forces at contacts and (increments of) relative displacements is through the contact constitutive relation. Since very small strains are considered, it is assumed that:

- The contact topology is fixed ("cemented contacts"), once a certain configuration with a certain coordination number has been chosen. Thus, contact creation and disruption are not taken into account. Changes in contact orientation are also ignored.

- Interparticle Coulomb friction is ignored in the following, since the number of interparticle contacts where Coulomb friction is fully mobilised ("sliding contacts") is very small in the initial isotropic state. Alternatively, one could state that the case of an infinite interparticle friction is considered.

- The contact elastic properties are constant: there is no variation in stiffness at contacts, contrary to the case with a non-linear elastic contact constitutive relation (such as a Hertzian-type).

Hence a simple linear elastic contact constitutive relation is employed here

$$
f_{i}^{c}=S_{i j}^{c} \Delta_{j}^{c} \quad S_{i j}^{c}=k_{n} n_{i}^{c} n_{j}^{c}+k_{t} t_{i}^{c} t_{j}^{c}
$$


where $S_{i j}^{c}$ is the contact stiffness matrix, $k_{n}$ and $k_{t}$ are the (constant) stiffnesses in normal and tangential directions of the (two-sided) springs.

The relations between particle properties and the contact stiffnesses $k_{n}$ and $k_{t}$ is studied in the field of contact mechanics (see for example Johnson, 1985). The ratio $k_{t} / k_{n}$ is related to the Poisson ratio of the particles (Johnson, 1985). In many studies the case $k_{t}=0$ is considered, when for disks interparticle forces are central and particle rotations are all zero. In the case $k_{t} / k_{n}=1$, force $f_{i}^{c}$ and relative displacement $\Delta_{i}^{c}$ at contacts are co-linear. The influence of the microscopic stiffness ratio $k_{t} / k_{n}$ on the macroscopic Poisson ratio has been studied by Bathurst $\&$ Rothenburg $(1988 \mathrm{a}, \mathrm{b})$ and Rothenburg et al. (1991).

The process of contact creation and disruption is generally not reversible. Only by fixing the contact topology and by ignoring the frictional Coulomb limit, can true reversible elastic behaviour be studied (Calvetti et al., 2002).

\subsection{Minimum potential energy principle}

Variational principles for continuous elastic solids are well established (for example, Hill, 1950; Washizu, 1968). Analogous discrete variational principles for granular materials have been formulated by Kruyt \& Rothenburg $(2002,2004)$ for systems with non-rotating and rotating particles, respectively. The discrete minimum potential energy principle reported by Kruyt \& Rothenburg (2004) is summarised here, as it will be used subsequently.

At the boundary $B$ of the system the displacements are prescribed. Consider two displacement and rotation fields $\left\{U_{i}^{p}, \Omega^{p}\right\}$ and $\left\{U_{i}^{*} p, \Omega^{*} p\right.$, which both satisfy the displacement boundary conditions on $B$. These displacement and rotation fields have associated relative displacement fields $\left\{\Delta_{i}^{c}\right\}$ and $\left\{\Delta_{i}^{*}\right\}$, according to Eq. (3). The corresponding contact force fields $\left\{f_{i}^{c}\right\}$ and $\left\{f_{i}^{* c}\right\}$ are determined from the contact constitutive relation Eq. (4). By definition, the contact force field $\left\{f_{i}^{\varepsilon}\right\}$ satisfies all equilibrium conditions Eq. (1), contrary to the contact force field $\left\{f_{i}^{* c}\right\}$.

The discrete minimum potential energy principle is

$$
\Pi\left(\left\{U_{i}^{p}, \Omega^{p}\right\}\right) \equiv \frac{1}{2 A} \sum_{c \in C} f_{i}^{c} \Delta_{i}^{c} \leq \frac{1}{2 A} \sum_{c \in C}{f_{i}^{*}}^{*} \Delta_{i}^{* c} \equiv \Pi\left(\left\{U_{i}^{* p}, \Omega^{* p}\right\}\right)
$$

This energy principle states that, among all displacement and rotation fields $\left\{U_{i}^{* p}, \Omega^{* p}\right\}$, the true displacement and rotation field $\left\{U_{i}^{p}, \Omega^{p}\right\}$, whose contact force field $\left\{f_{i}^{c}\right\}$ satisfies the equilibrium conditions Eq. (1), makes the potential energy density $\Pi$ an absolute minimum. 


\subsection{Predictions of moduli: energy-based vs. stress-based approaches}

Consider the case where the displacement $U_{l}^{\beta}$ of the particles $\beta$ at the boundary $B$ conforms to the prescribed uniform (increment of the) displacement gradient $\alpha_{i j} \equiv \partial U_{i} / \partial x_{j}$, i.e. $U_{i}^{\beta}=\alpha_{i j} X_{j}^{\beta}$. The (increment of the) strain tensor $\varepsilon_{i j}$ equals the symmetric part of the displacement gradient tensor

$$
\varepsilon_{i j}=\frac{1}{2}\left(\alpha_{i j}+\alpha_{j i}\right)
$$

The energy density $\Pi\left(\left\{U_{i}^{p}, \Omega^{p}\right\}\right)$ of the true displacement and rotation field $\left\{U_{i}^{p}, \Omega^{p}\right\}$ will now be expressed in terms of the strain tensor $\varepsilon_{i j}$ and the fourth-order elastic stiffness tensor $L_{i j k l}$, which relates stress and strain through

$$
\sigma_{i j}=L_{i j k l} \varepsilon_{k l}
$$

According to the virtual work principle (Kruyt \& Rothenburg, 2002, 2004) and the assumed boundary displacements, we find

$$
\sum_{c \in C} f_{i}^{c} \Delta_{i}^{c}=\sum_{\beta \in B} f_{i}^{\beta} U_{i}^{\beta}=\left(\sum_{\beta \in B} f_{i}^{\beta} X_{j}^{\beta}\right) \alpha_{i j}=A \sigma_{i j} \alpha_{i j}=A \sigma_{i j} \varepsilon_{i j}
$$

since the stress tensor $\sigma_{i j}$ is given by $\sigma_{i j}=(1 / A) \sum_{\beta \in B} f_{l}^{\beta} X_{j}^{\beta}$ (Kruyt \& Rothenburg, 1996) and the stress tensor is symmetric due to the moment equilibrium equations. Thus, the energy density, Eq. (5), of the true displacement and rotation field is given by $\Pi\left(\left\{U_{i}^{p}, \Omega^{p}\right\}\right)=(1 / 2) \varepsilon_{i j} L_{i j k l} \varepsilon_{k l}$.

For the case considered of isotropic assemblies, the elastic stiffness tensor $L_{i j k l}$ is isotropic. It is then described by the bulk modulus $K$ and the shear modulus $G$. These can be determined by considering the two loading paths given in Eq. (18). For isotropic compression, the stress tensor is given by $\sigma_{i j}=2 K \varepsilon_{i j}$, while for pure shearing $\sigma_{i j}=2 G \varepsilon_{i j}$. For conciseness of notation, this is expressed as $\sigma_{i j}=2 M \varepsilon_{i j}$. Here the elastic modulus $M$ is either the bulk modulus or the shear modulus, depending on the loading path. In terms of this elastic modulus $M$, the energy density Eq. (5) of the true displacement and rotation field is given by $\Pi\left(\left\{U_{i}^{p}, \Omega^{p}\right\}\right)=M \varepsilon_{i j} \varepsilon_{i j}$.

By evaluating the energy density $\Pi\left(\left\{U_{i}^{*} p, \Omega^{*}{ }^{*}\right\}\right)$ of a displacement and rotation field $\left\{U_{i}^{*}, \Omega^{*} p\right\}$ whose associated contact force field $\left\{f_{i}^{* c}\right\}$ does not satisfy all equilibrium conditions Eq. (1), a rigorous upper bound for the modulus $M$ is obtained

$$
M \varepsilon_{i j} \varepsilon_{i j}=\Pi\left(\left\{U_{i}^{p}, \Omega^{p}\right\}\right) \leq \Pi\left(\left\{U_{i}^{*} p \Omega^{*} p\right\}\right)
$$

This is called the energy-based approach for obtaining a prediction of the modulus $M$, based on the displacement and rotation field $\left\{U_{i}^{*}, \Omega^{*} p\right\}$. Note that different displacement and rotation fields 
yield different bounds for the moduli.

Alternatively, from a displacement and rotation field $\left\{U_{i}^{*} p, \Omega^{*}\right.$ \} with associated contact force field $\left\{f_{i}^{*}{ }^{c}\right\}$, a stress tensor $\sigma_{i j}^{*}$ can be determined according to Eq. (2). From this stress tensor the modulus $M$ can be computed from $\sigma_{i j}^{*}=2 M \varepsilon_{i j}$, since the displacement gradient $\alpha_{i j}$, and hence the strain tensor $\varepsilon_{i j}$, has been prescribed. This is called the stress-based approach.

The derivation of Eq. (2) is based on the equilibrium conditions Eq. (1). The contact force field $\left\{f_{i}^{*}\right\}$ generally does not satisfy all equilibrium conditions. Thus, the stress tensor $\sigma_{i j}^{*}$, obtained by using Eq. (2) $\sigma_{i j}^{*}=(1 / A) \sum_{c \in C} f_{i}^{* c} l_{j}^{c}$, is (strictly) not a true stress tensor.

For the true displacement and rotation field $\left\{U_{i}^{p}, \Omega^{p}\right\}$, the energy-based and the stress-based approaches yield the same modulus.

\section{Local neighbourhood approach}

\subsection{Basic idea}

To obtain a prediction of the elastic modulus, a displacement and rotation field $\left\{U_{i}^{*}, \Omega^{*} p\right.$ is required. The basic idea in the proposed approach is to determine these fields from the solution of the force and moment equilibrium equations of neighbourhoods, or small sub-assemblies. These equations are solved with prescribed displacements and rotations, according to the imposed displacement gradient, of the outer particles of these sub-assemblies.

For a specified particle $p$, this particle together with its direct neighbours, i.e. those particles with which it is in contact, form a first-order sub-assembly. For a particle $p$, its second-order sub-assembly consists of the particles in the first-order sub-assembly, together with the neighbours of the outer particles in the first-order sub-assembly (but excluding particles that already belong to the first-order sub-assembly). This can easily be generalised to larger sub-assembly orders (or sizes), as illustrated in Figure 2.

For each particle $p$, its corresponding sub-assembly is determined. With prescribed displacements and rotations of the outer particles at the boundary of the sub-assembly (as indicated in Figure 2), the displacements and rotations of all inner particles are determined from the force and moment equilibrium equations. The formulation of these equilibrium equations is described in detail in Section 3.2. Only the displacements and rotation of particle $p$ are retained from this analysis. This procedure is followed for each particle independently. In the end, a displacement and rotation field $\left\{U_{i}^{*}, \Omega^{*}{ }^{p}\right\}$ is obtained. From these fields, predictions of the moduli are determined, as described in Section 2.2.

This approach has already been used by Kruyt \& Rothenburg $(2002,2004)$ using first-order sub- 
First-order sub-assembly; $m=1$

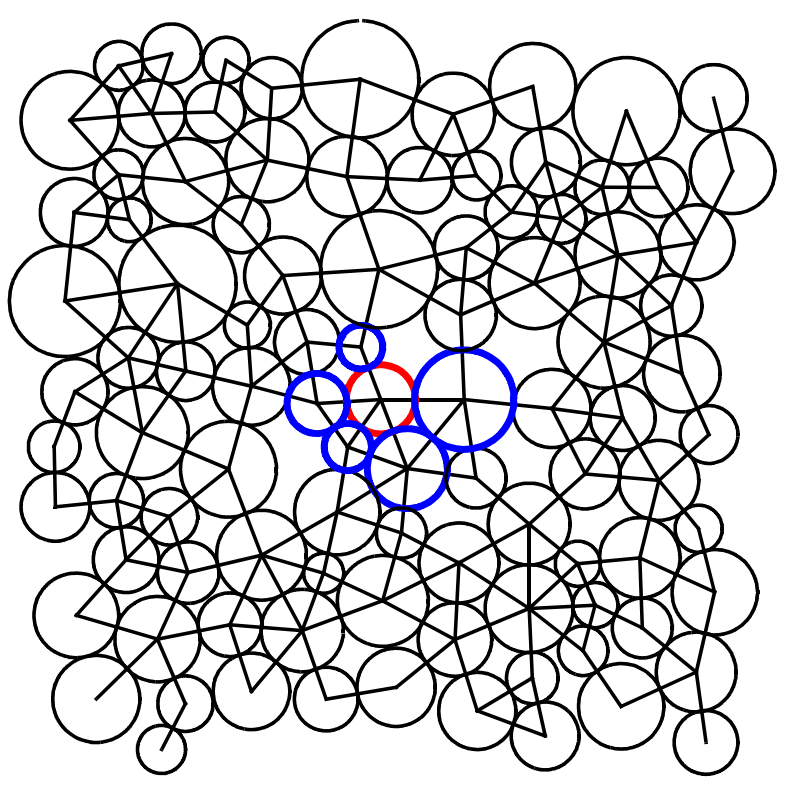

Third-order sub-assembly; $m=3$

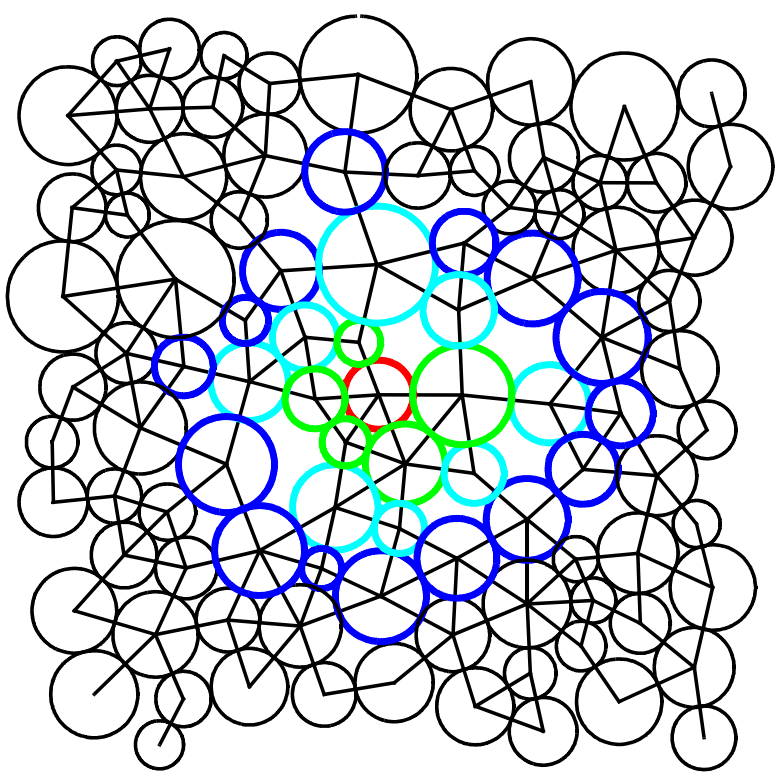

Second-order sub-assembly; $m=2$

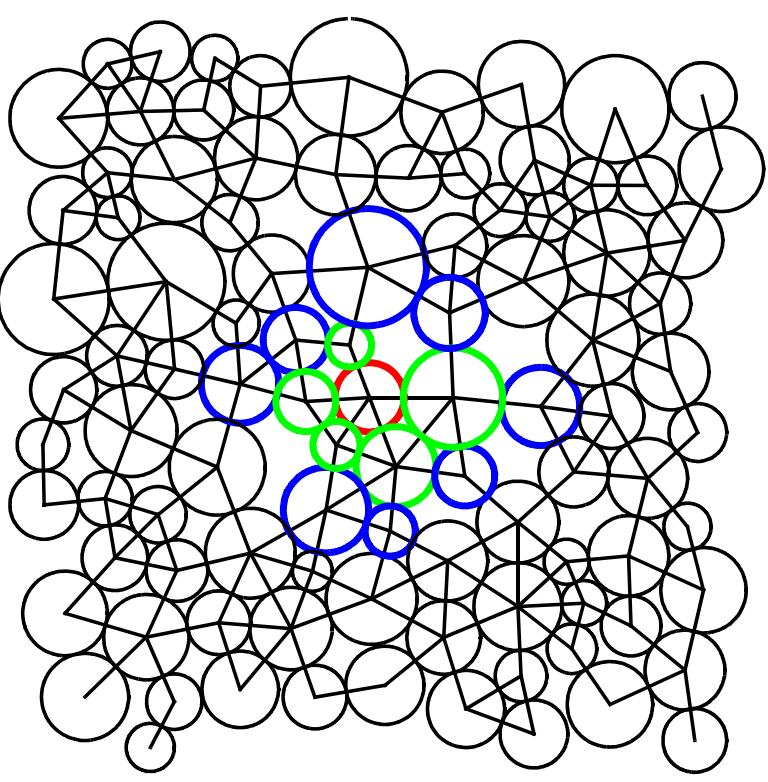

Fourth-order sub-assembly; $m=4$

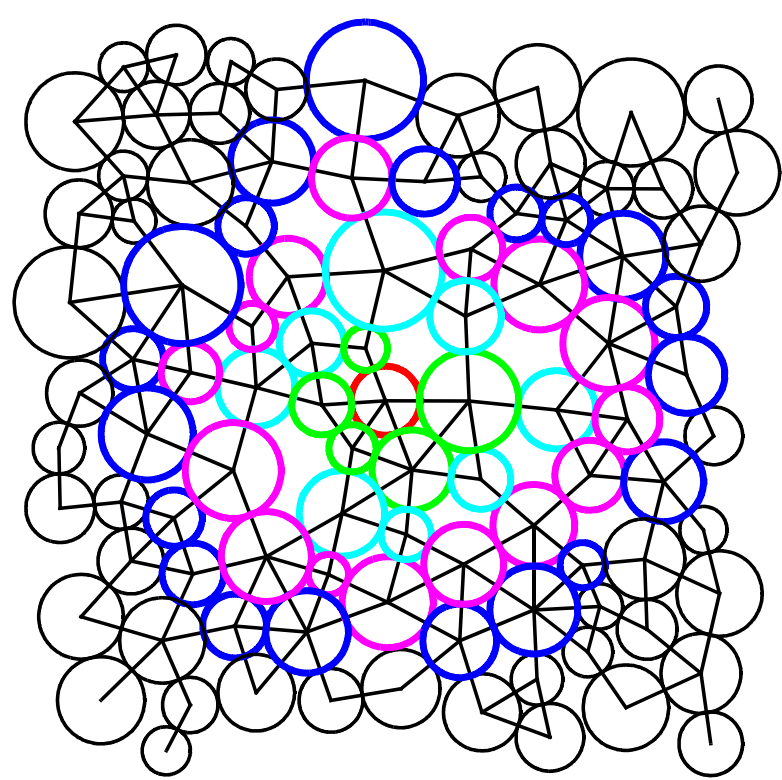

Figure 2. Sketch of sub-assemblies for a specified central particle shown in red. Top left: first-order, top right: second-order, bottom left: third-order, bottom right: fourth-order sub-assembly. The outer particles of the sub-assembly, shown in dark blue, are assigned displacements and rotations that conform to the meanfield deformation. The displacements and rotations of the inner particles are determined from the equilibrium equations. Particles shown in black are ignored in the analysis of the sub-assembly for the red particle. Contacts between particles are denoted by lines connecting their centres. Only part of the full assembly is shown. 
assemblies. Here it is generalised by considering larger sub-assemblies.

\subsection{Solution of equilibrium equations for sub-assemblies}

Now it is described in detail how the unknown displacements and rotations of the particles inside the sub-assembly are obtained from the equilibrium conditions Eq. (1) for these particles. This solution process consists of three steps:

- definition of fluctuations, relative to the mean field, in particle displacements and rotations;

- definition of the "element matrix", in FEM terminology, that specifies the interaction between two particles in contact;

- formulation and solution of the system of linear equilibrium equations, in terms of the fluctuations, for the sub-assembly.

\subsubsection{Fluctuations}

The displacements, $U_{i}^{p}$, and rotations, $\Omega^{p}$, of particles are divided into contributions due to the imposed displacement gradient $\alpha_{i j}$ and fluctuations

$$
U_{i}^{p}=\alpha_{i j} X_{j}^{p}+u_{i}^{p} \quad \Omega^{p}=\frac{1}{2}\left(\alpha_{21}-\alpha_{12}\right)+\omega^{p}
$$

where $\left(\alpha_{21}-\alpha_{12}\right) / 2$ is the average system rotation corresponding to the imposed displacement gradient $\alpha_{i j}$, while $u_{i}^{p}$ and $\omega^{p}$ are the displacement and rotation fluctuation of particle $p$, respectively. As before $X_{i}^{p}$ is the position vector of the centre of particle $p$. Effectively, Eq. (10) represents a change of variables from $\left\{U_{i}^{p}, \Omega^{p}\right\}$ to $\left\{u_{i}^{p}, \omega^{p}\right\}$. While the particle displacements are, on average, position-dependent, the fluctuations are zero on average over all particles.

In the uniform strain assumption, all displacement and rotation fluctuations are taken equal to zero, i.e. $u_{i}^{p} \equiv 0$ and $\omega^{p} \equiv 0$. Hence the corresponding relative displacements $\Delta_{i}^{c, \alpha}$ are given by (see Eq. (3))

$$
\Delta_{i}^{c, \alpha}=\varepsilon_{i j} l_{j}^{c}
$$

where $l_{i}^{c}$ is the branch vector at contact $c$ as defined in Section 2 . The superscript $\alpha$ is present in the quantity $\Delta_{i}^{c, \alpha}$, since it depends on the imposed displacement gradient $\alpha_{i j}$.

\subsubsection{Element matrix}

A contact between two particles $p$ and $q$ can be considered as a "finite element" (with two nodes). The interaction between the particles is specified by the "element matrix" in FEM terminol- 
ogy, see for example Zienkiewicz \& Taylor (1987). The element matrix relates the generalised displacements at the contact to the generalised load at the contact.

The generalised contact displacement vector $\boldsymbol{W}^{p q}$ (of length 6) and the generalised contact load vector $\boldsymbol{F}^{p q}$ (of length 6 ) of a contact $c \equiv p q$ are defined by

$$
\begin{aligned}
\boldsymbol{W}^{p q} & =\left[\begin{array}{llllll}
u_{1}^{p} & u_{2}^{p} & \omega^{p} & u_{1}^{q} & u_{2}^{q} & \omega^{q}
\end{array}\right]^{T} \\
\boldsymbol{F}^{p q} & =\left[\begin{array}{llllll}
f_{1}^{p q} & f_{2}^{p q} & R^{p} \cdot f_{t}^{p q} & f_{1}^{q p} & f_{2}^{q p} & R^{q} \cdot f_{t}^{q p}
\end{array}\right]^{T}
\end{aligned}
$$

where $f_{t}^{p q}$ is the tangential component of the force vector $f_{i}^{p q}$ exerted by particle $q$ on particle $p$.

The generalised contact load vector $\boldsymbol{F}^{p q}$ and the generalised contact displacement vector $\boldsymbol{W}^{p q}$ are related by

$$
\boldsymbol{F}^{p q}=-\boldsymbol{K}^{p q} \cdot \boldsymbol{W}^{p q}
$$

The symmetrical 6 by 6 element matrix $\boldsymbol{K}^{p q}$ corresponding to the contact constitutive relation Eq. (4) is given by

$$
\boldsymbol{K}^{p q}=\tau\left(\theta^{p q}\right)^{T} \cdot\left[\begin{array}{cccccc}
k_{n} & 0 & 0 & -k_{n} & 0 & 0 \\
0 & k_{t} & R^{p} k_{t} & 0 & -k_{t} & R^{q} k_{t} \\
0 & R^{p} k_{t} R^{p} R^{p} k_{t} & 0 & -R^{p} k_{t} & R^{p} R^{q} k_{t} \\
-k_{n} & 0 & 0 & k_{n} & 0 & 0 \\
0 & -k_{t} & -R^{p} k_{t} & 0 & k_{t} & -k_{t} R^{q} \\
0 & R^{q} k_{t} R^{p} R^{q} k_{t} & 0 & -k_{t} R^{q} & R^{q} R^{q} k_{t}
\end{array}\right] \cdot \tau\left(\theta^{p q}\right)
$$

Here the symmetrical 6 by 6 transformation matrix $\tau\left(\theta^{p q}\right)$, from local $(n, t)$-variables at the contact to Cartesian variables, is given by

$$
\tau(\theta)=\left[\begin{array}{cccccc}
\cos \theta & -\sin \theta & 0 & 0 & 0 & 0 \\
\sin \theta & \cos \theta & 0 & 0 & 0 & 0 \\
0 & 0 & 1 & 0 & 0 & 0 \\
0 & 0 & 0 & \cos \theta & -\sin \theta & 0 \\
0 & 0 & 0 & \sin \theta & \cos \theta & 0 \\
0 & 0 & 0 & 0 & 0 & 1
\end{array}\right]
$$




\subsubsection{Formulation of equilibrium equations for a sub-assembly}

The sub-assembly represents a structure, with kinematic boundary conditions according to the imposed displacement gradient for the outer particles of the sub-assembly. The connectivity of the nodes (particles) is determined by the contacts. The interaction between the nodes (particles) is expressed by the element matrix given in Eq. (14). By "assembling" (see the Zienkiewicz \& Taylor, 1987) the global system of equilibrium equations for the small sub-assembly from the element matrices (contacts), the linear system of equations to be solved for the fluctuations is obtained (formally)

$$
\left(\sum_{c \in C(p ; m)} \boldsymbol{T}^{c T} \cdot \boldsymbol{K}^{c} \cdot \boldsymbol{T}^{c}\right) \cdot \boldsymbol{V}^{p}=\sum_{c \in C(p ; m)}\left(\boldsymbol{T}^{c T} \cdot \boldsymbol{K}^{c} \cdot \boldsymbol{U}^{\alpha, c}\right)
$$

Here $V^{p}$ is the vector with all unknown fluctuation displacements and fluctuation rotations within the sub-assembly for particle $p$; the length of $\boldsymbol{V}^{p}$ is $N(p ; m)$. The sum is over all contacts $c$ within the set of contacts $C(p ; m)$ of the sub-assembly for particle $p$ of order $m$. The matrix $\boldsymbol{T}^{c}$ (of size 6 by $N(p ; m))$ accounts for the extraction of the global degrees of freedom within the sub-assembly to the degrees of freedom corresponding to contact $c$. Note that each row of this matrix contains only a single element equal to one, while the other elements are all zero. The matrix $\boldsymbol{T}^{c T}$, the transpose of $\boldsymbol{T}^{c}$, accounts for the opposite transformation, going from local contact quantities to the corresponding global quantity in the vector $\boldsymbol{V}^{p}$. The vector $\boldsymbol{U}^{\alpha, c}$ (of length 6) contains the displacements and rotations at contact $c$ according to the average displacement gradient $\boldsymbol{\alpha}$

$$
\boldsymbol{U}^{\alpha, c}=\left[\left(\boldsymbol{\alpha} \cdot \boldsymbol{X}^{T(c)}\right)_{1}\left(\boldsymbol{\alpha} \cdot \boldsymbol{X}^{T(c)}\right)_{2} \frac{1}{2}\left(\alpha_{21}-\alpha_{12}\right)\left(\boldsymbol{\alpha} \cdot \boldsymbol{X}^{H(c)}\right)_{1}\left(\boldsymbol{\alpha} \cdot \boldsymbol{X}^{H(c)}\right)_{2} \frac{1}{2}\left(\alpha_{21}-\alpha_{12}\right)\right]^{T}
$$

Here $H(c)$ and $T(c)$ give the 'head' (second) and 'tail' (first) particles involved in contact $c$ and $\left(\boldsymbol{\alpha} \cdot \boldsymbol{X}^{H(c)}\right)_{1}$ gives the first component of the vector $\boldsymbol{\alpha} \cdot \boldsymbol{X}^{H(c)}$ (of length 2) and other terms are defined analogously. As before, $\boldsymbol{X}^{p}$ gives the position vector of the centre of particle $p$. The position vector $\boldsymbol{X}^{H(c)}$ is conveniently expressed as $\boldsymbol{X}^{H(c)}=\boldsymbol{X}^{T(c)}+\boldsymbol{l}^{c}$ (where $\boldsymbol{l}^{c}$ is the branch vector of contact $c$, see Figure 1) to account for 'wrap-around' of position vectors in the periodic systems studied here. In essence, the right-hand side of Eq. (16) represents the loading of the particles due to the imposed mean field $\boldsymbol{\alpha}$ (see also, Kruyt \& Rothenburg, 2002, 2004).

For a specified particle $p$ and order of the sub-assembly $m$, the vector $\boldsymbol{V}^{p}$ is computed from the equilibrium equations Eq. (16) for the inner particles. Only the fluctuation displacement $u_{i}^{p}$ and the rotation $\omega^{p}$ of particle $p$ are retained. This procedure is executed for all particles consecutively. Note that these computations are uncoupled. In this manner an approximation $\left\{U_{i}^{*} p, \Omega^{*} p\right.$ of the 
true displacement and rotation field $\left\{U_{i}^{p}, \Omega^{p}\right\}$ is constructed.

The displacements of particles at the boundary $B$ are not changed, since these are prescribed according to the mean field. Since the average displacement gradient $\alpha_{i j}$ is determined by the displacements at the boundary $\alpha_{i j}=(1 / A) \int_{B} U_{i} n_{j} d B$, as follows from Gauss theorem (see also Kruyt \& Rothenburg, 1996), the displacement and rotation fields $\left\{U_{i}^{*} p, \Omega^{*} p\right.$ are always consistent with the prescribed displacement gradient $\alpha_{i j}$.

\section{DEM simulations}

Discrete Element Method simulations, as proposed by Cundall \& Strack (1979), have been performed with two loose assemblies of 50,000 disks from a fairly wide lognormal particle-size distribution, for which the ratio of the standard deviation over the mean equals 0.25 . The coordination numbers of the two assemblies are $\Gamma=3.53$ and $\Gamma=4.08$. The corresponding packing fractions $\eta$, i.e. the area occupied by the particles divided by the area of the assembly (including voids), are $\eta=0.756$ and $\eta=0.829$, respectively. Initially, the periodic box is square with a length of about 200 times the average particle diameter. The assemblies are isotropic, as shown by the contact distribution function $E(\theta)$ (defined in Section 2) in Figure 3. The percentage of "rattlers", i.e. floating particles without contacts, is $8 \%$ and $2 \%$ for $\Gamma=3.53$ and $\Gamma=4.08$, respectively. Note that these coordination numbers are those obtained after removal of rattlers.

In the DEM simulations used for creating these two initial assemblies, contact creation and disruption are taken into account (contrary to the DEM simulations reported subsequently). The assembly with $\Gamma=3.53$ has been obtained by a succession of frictionless and frictional isotropic compressions. The assembly with $\Gamma=4.08$ has been obtained by the isotropic compression of a frictionless gas, i.e. with $k_{t} \equiv 0$. In both cases a specified pressure has been employed as boundary condition. Periodic boundaries have been used in all DEM simulations to minimize boundary effects.

Starting from these two initial assemblies, DEM simulations with fixed contact topology have been performed. Thus, contact creation and disruption are not taken into account. The linear elastic constitutive relation (4) is used, where frictional limits are ignored. Various values for the stiffness ratio $k_{t} / k_{n}$ have been considered, for each of the two assemblies, in order to systematically investigate the influence of $k_{t} / k_{n}$, with exactly the same contact geometry.

The assemblies were subjected to the loading paths for compression $\alpha_{i j}^{K}$ and shear $\alpha_{i j}^{G}$, where these displacement gradients are given by 


$$
\alpha_{i j}^{K}=\alpha_{\text {mag }}\left[\begin{array}{ll}
1 & 0 \\
0 & 1
\end{array}\right] \quad \alpha_{i j}^{G}=\alpha_{\text {mag }}\left[\begin{array}{cc}
-1 & 0 \\
0 & 1
\end{array}\right]
$$

where $\alpha_{\text {mag }}$ is a magnitude of the imposed displacement gradient. Note that the value $\alpha_{\text {mag }}$ is not important, since the adopted model results in linear governing equations.

In the stress-based approach for estimating the elastic moduli (see Section 5.1), the macroscopic bulk $K$ and shear modulus $G$ are determined from

$$
\begin{aligned}
& \sigma_{11}+\sigma_{22}=2 K\left(\varepsilon_{11}+\varepsilon_{22}\right) \\
& \sigma_{11}-\sigma_{22}=2 G\left(\varepsilon_{11}-\varepsilon_{22}\right)
\end{aligned}
$$

Alternatively, the macroscopic elastic moduli can be expressed in terms of macroscopic Young's modulus $Y$ and Poisson ratio $v$. In the two-dimensional case considered here, $Y$ and $v$ are related to the bulk and shear moduli by

$$
v=\frac{K-G}{K+G} \quad Y=\frac{4 K G}{K+G}
$$

According to the uniform strain assumption Eq. (11), the bulk modulus $K^{\varepsilon}$ and the shear modulus $G^{\varepsilon}$ are given by (see Kruyt \& Rothenburg, 1996, 1998)
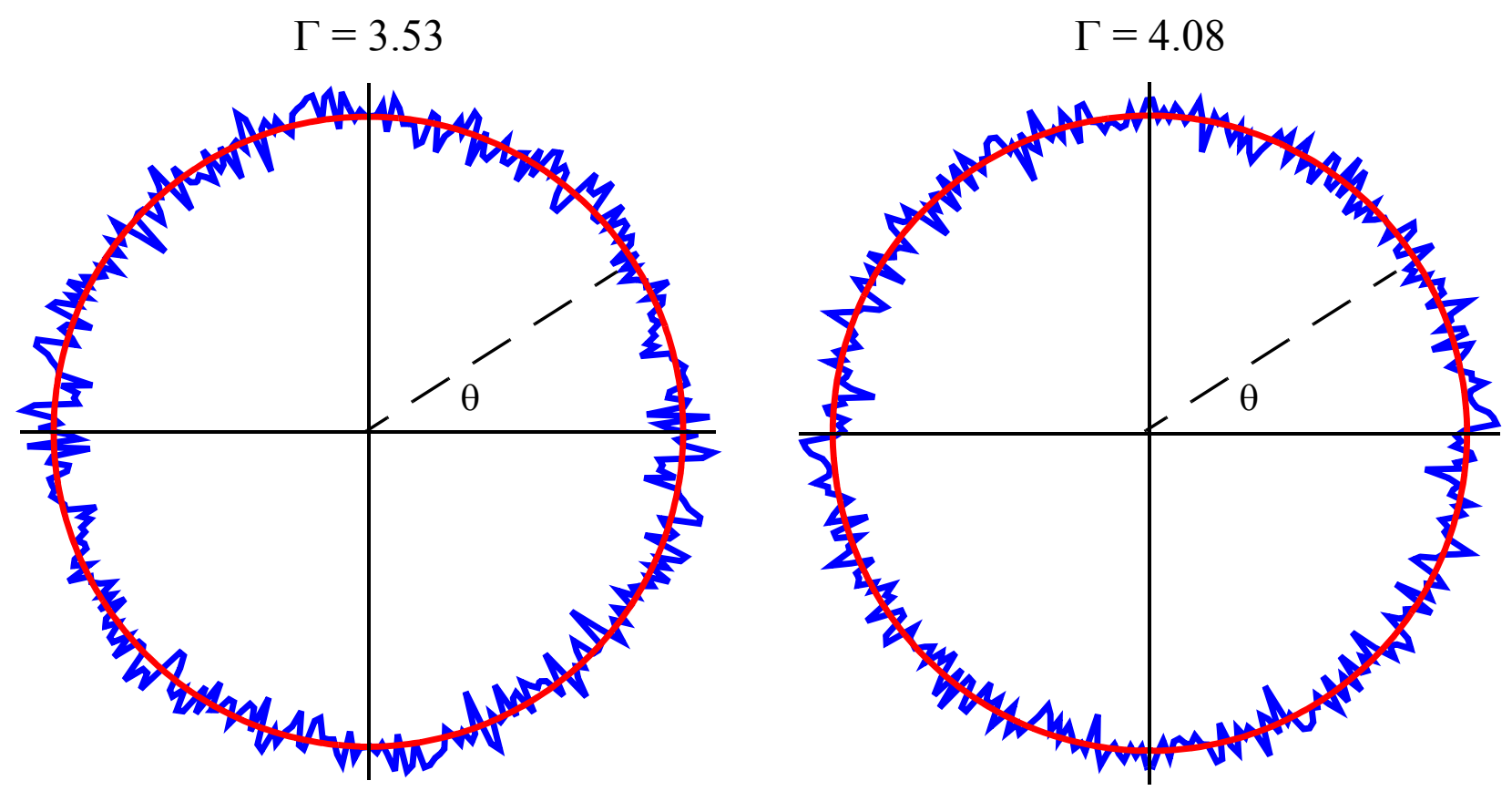

Figure 3. Polar plots of the contact distribution function $E(\theta)$ for assemblies with coordination numbers $\Gamma=3.53$ (left) and $\Gamma=4.08$ (right); in blue: actual value based on bins with width of $1^{\circ}$, in red: isotropic value $E(\theta)=1 /(2 \pi)$. 


$$
\frac{K^{\varepsilon}}{k_{n}}=\frac{c_{A}}{4} \overline{l_{n}^{2}} \quad \frac{G^{\varepsilon}}{k_{n}}=\frac{c_{A}}{8}\left[1+\frac{k_{t}}{k_{n}}\right] \overline{l_{n}^{2}}
$$

where $c_{A}$ is the contact number density, i.e. the number of contacts per unit area, and $\overline{l_{n}^{2}}$ is the average over all contacts of the length of the branch vector squared. The evolution of the elastic stiffness tensor according to the uniform strain assumption in biaxial deformation has been studied by Luding (2004).

The length scales of a granular assembly at which continuum elasticity theory is applicable have been studied by Goldenberg \& Goldhirsch (2002) and Goldhirsch \& Goldenberg (2002). Kuhn \& Bagi (2009) have shown that the average initial elastic modulus is hardly influenced by the system size, i.e. the ratio between average particle radius and size of the periodic box. It must be noted that these authors use a different contact constitutive relation that allows for contact disruption and creation, whereas here contacts are fixed (“cemented contacts"). Somfai et al. (2005) have shown, how-
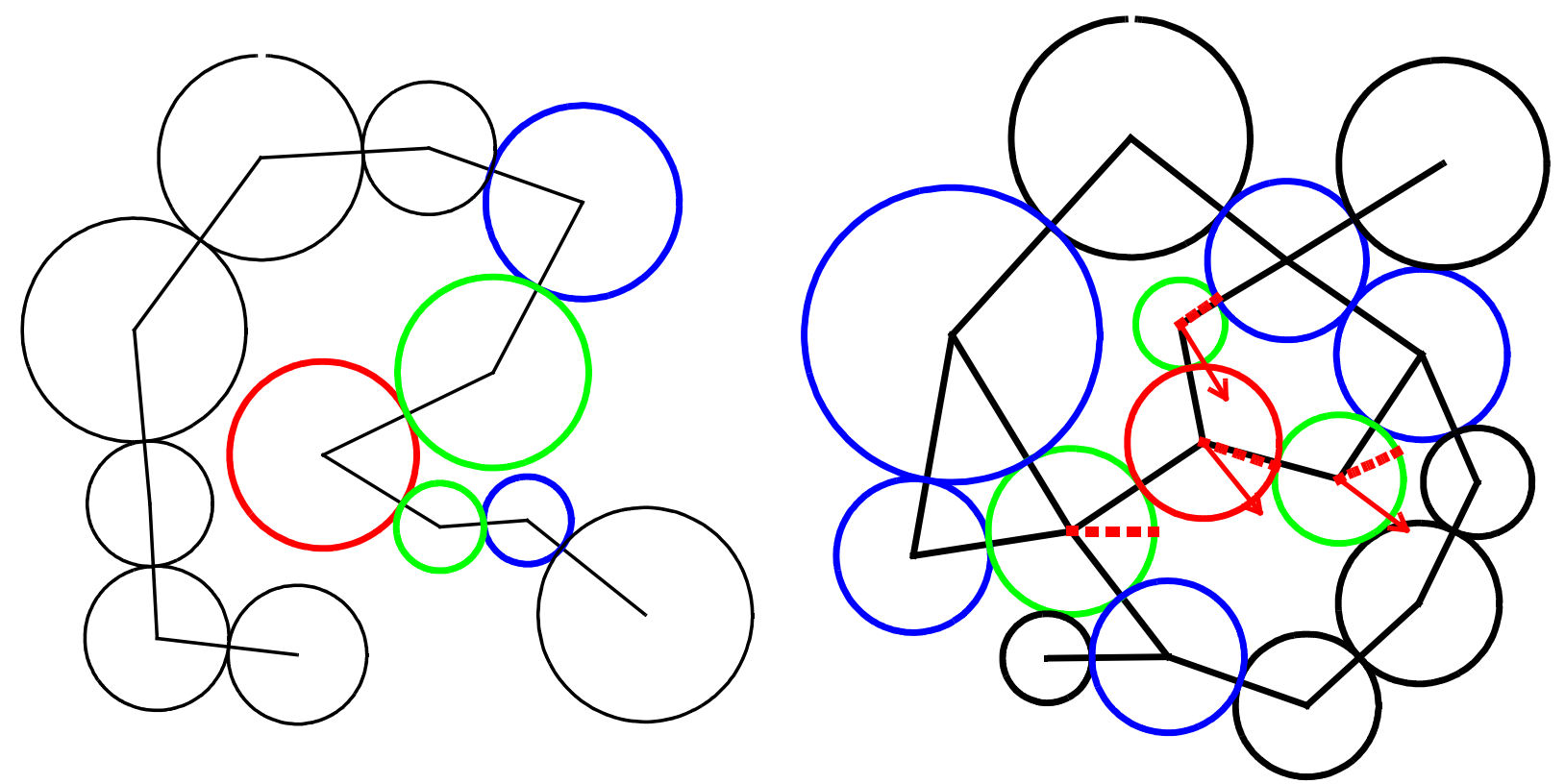

Figure 4. Sketch of near-singular configurations. Left: Singular "chain" of unstable red and green particles that each have two contacts. Right: Near-singular configuration for the red particle with sub-assembly order $m=2$. The condition number, i.e. the ratio between the largest and the smallest eigenvalue of the matrix of the system of equations in Eq. (16), is 1300. The displacement and rotation field corresponding to the smallest eigenvalue of this matrix is indicated by vectors for the displacements of the particles and by dotted lines for the rotation of the particles, relative to the horizontal. This eigenmode closely corresponds to rolling at the contacts. 
ever, that sound propagation characteristics are more sensitive to system size.

Visualisation of the displacement fields from the DEM simulations showed some very large particle displacements fluctuations. These "outliers", in statistical investigations of the displacement and rotation fluctuation fields, are due to singular and near-singular configurations. Instead of eliminating these outliers, based on an arbitrary magnitude criterion, some "filtering" of contacts has been performed on the assemblies to remove such singular and near-singular configurations. The first employed criterion is that each particle should have at least two contacts. The second criterion is that no "chains" of particles (see Figure 4 on the left) with two contacts each are allowed. Corresponding contacts are removed and some particles become "rattlers". The third criterion is that of the particles that have three contacts, no more than one of the corresponding particles may have two contacts. This third criterion rules out configurations as sketched in Figure 4 on the right. Thus, the contacts of the red particle are removed and the red particle becomes a rattler. Consequently, the top and right green particles also become rattlers, as they have fewer than two contacts. Rattlers are ignored in all subsequent analyses. By employing these criteria, the number of contacts decreases by $1.5 \%$ for $\Gamma=3.53$ and by $0.3 \%$ for $\Gamma=4.08$. Note that these values for the coordination number are obtained after removal of "rattlers" and after "filtering" out unstable contacts.

The DEM simulations have been performed such that the maximum resultant force and moment (i.e. the deviations from equilibrium), measured relative to the average normal force (and the average particle radius for the moment equilibrium equation) acting on each particle, is less than $0.1 \%$.

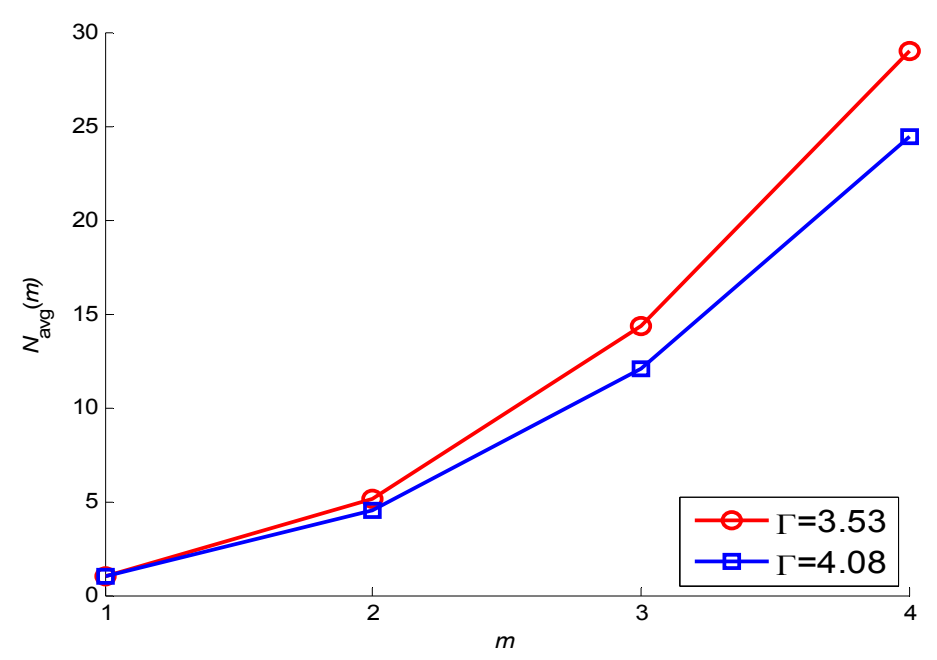

Figure 5. Average number of particles $N_{\text {avg }}(m)$ inside the sub-assembly whose displacements and rotations are computed, for various sub-assembly orders $m$ and coordination numbers $\Gamma$. The increase of $N_{\text {avg }}(m)$ is approximately quadratic in $m$. 


\section{Results}

In this section the results for the predictions of the elastic moduli are given. Firstly, the average number of particles inside the sub-assemblies, whose displacements and rotations have to be computed, is shown in Figure 5 for various sub-assembly orders $m$. This number increases rapidly (almost quadratically) with $m$.

\subsection{Energy-based vs. stress-based approaches}

Predictions of the moduli, based on approximate displacement and rotation fields $\left\{U_{i}^{*} p, \Omega^{*} p\right\}$, can be obtained in two ways (see Section 2.2): with the energy-based and the stress-based approaches. These predictions for the shear modulus are compared for various sub-assembly orders $m$ in Figure 6.

With increasing sub-assembly order $m$, both approaches give a more accurate prediction of the true shear modulus from the DEM simulation. For the case shown in Figure 6 all predictions lie above the true value. That this holds for the energy-based predictions follows rigorously from the minimum potential energy principle of Section 2.1. For the stress-based predictions this can not be shown. Indeed, for very small stiffness ratios $k_{t} / k_{n}$ the stress-based prediction may drop below the true DEM-value. Generally, the stress-based predictions are closer to the true value than the energybased predictions, see Figure 6. Therefore, the stress-based approach is adopted in the following.

\subsection{Predicted bulk and shear moduli}

The predictions for the bulk modulus $K$ and the shear modulus $G$ are shown in Figure 7 for the coordination numbers $\Gamma=3.53$ and $\Gamma=4.08$ for various values of the stiffness ratio $k_{t} / k_{n}$, together with the moduli according to the uniform strain assumption Eq. (21) and with the true moduli from the DEM simulations.

The moduli according to the uniform strain assumption differ strongly from the true moduli. This is especially the case for the shear modulus $G$ for the loosest assembly with $\Gamma=3.53$, where the average relative deviation is $140 \%$.

The magnitude of the deviations of the predictions from the true DEM-value decrease with increasing sub-assembly order $m$. The deviations are larger for the shear modulus $G$ than for the bulk modulus $K$. The deviations decrease with increasing coordination number $\Gamma$.

Figure 7 shows that the micromechanical predictions capture the dependence of the bulk modulus $G$ on the stiffness ratio $k_{t} / k_{n}$. Quantitatively, the bulk modulus is already well predicted for 
$m=2$ with average relative deviations of $8 \%$ and $4 \%$ for $\Gamma=3.53$ and $\Gamma=4.08$, respectively. For $m=3$, these average relative deviations are $4 \%$ and $3 \%$, while for $m=4$, these deviations are $2 \%$ and $2 \%$.

Figure 7 shows that the predictions capture the dependence of the shear modulus $G$ on the stiffness ratio $k_{t} / k_{n}$. Quantitatively, the shear modulus is already fairly well predicted for $m=2$ with average relative deviations of $38 \%$ and $13 \%$ for $\Gamma=3.53$ and $\Gamma=4.08$, respectively. For $m=3$, these average relative deviations are $16 \%$ and $6 \%$, while for $m=4$, these deviations are $2 \%$ and $2 \%$.

Note that for the lowest value of the stiffness ratio used, the stress-based prediction of the shear modulus falls slightly below the true DEM-value for $m=4$. Thus, these predictions do not form rigorous upper bounds to the moduli, contrary to the energy-based predictions.

\subsection{Influence of local vs. average contact stiffness matrix}

The equilibrium conditions Eq. (16) for the displacement and rotation fluctuations of the particles inside a sub-assembly involve the actual contact stiffness matrices of the particles. For possible simplifications of the current approach and, more importantly, for consideration in analytical approaches, it is interesting to investigate whether it is possible to replace the sum of the local con-

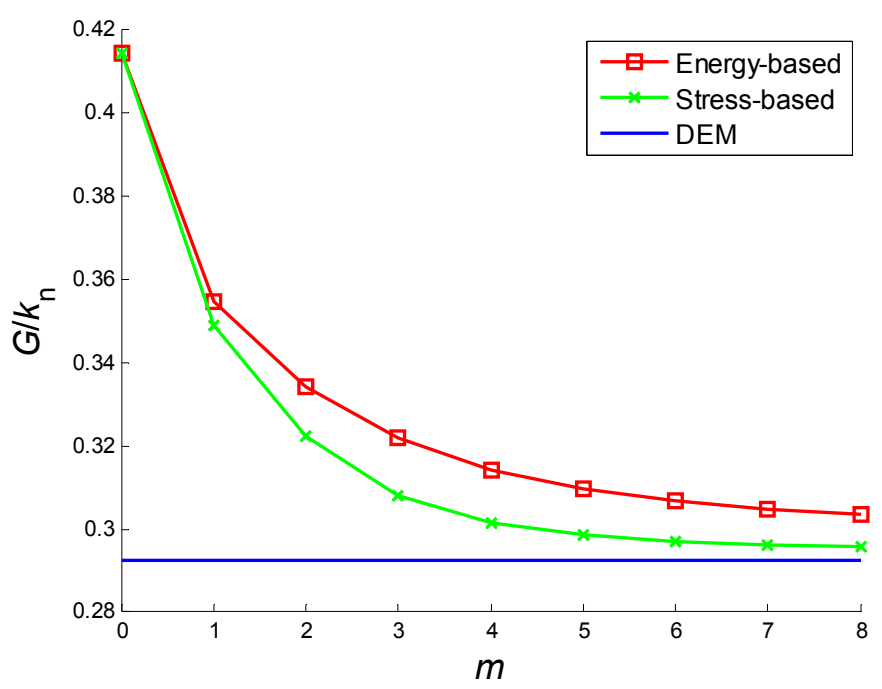

Figure 6. Predictions of the shear modulus $G$ for various sub-assembly orders $m$ for energy-based and stress-based approaches; coordination number $\Gamma=4.08$ and stiffness ratio $k_{t} / k_{n}=0.5$. The prediction for $m=0$ is the shear modulus according to the uniform strain assumption, Eq. (21). The blue line gives the result from the corresponding DEM simulation. 


\section{N.P. Kruyt \& I. Agnolin \& S. Luding \& L. Rothenburg}

tact stiffness matrices of the particles by representative averages without detrimental effects on the accuracy of the predictions. The local contact stiffness matrix is present in the element matrix representing the actual particle stiffness matrices on the left-hand side of Eq. (16) and in the right handside of Eq. (16) representing the resultant forces due to mean-field deformation.

For the matrix on the left-hand side of Eq. (16) this simplification amounts to replacing the sum of the local contact stiffness matrices by an average matrix. This is achieved by replacing $\sum_{q} K_{i j}^{p q}$ (where the sum is over the particles $q$ that are in contact with particle $p$ ) by the average $\Gamma_{p} \bar{K}_{i j}$, where $\Gamma_{p}$ is the number of contacts of particle $p$. The average stiffness matrix $\bar{K}_{i j}$ is defined by
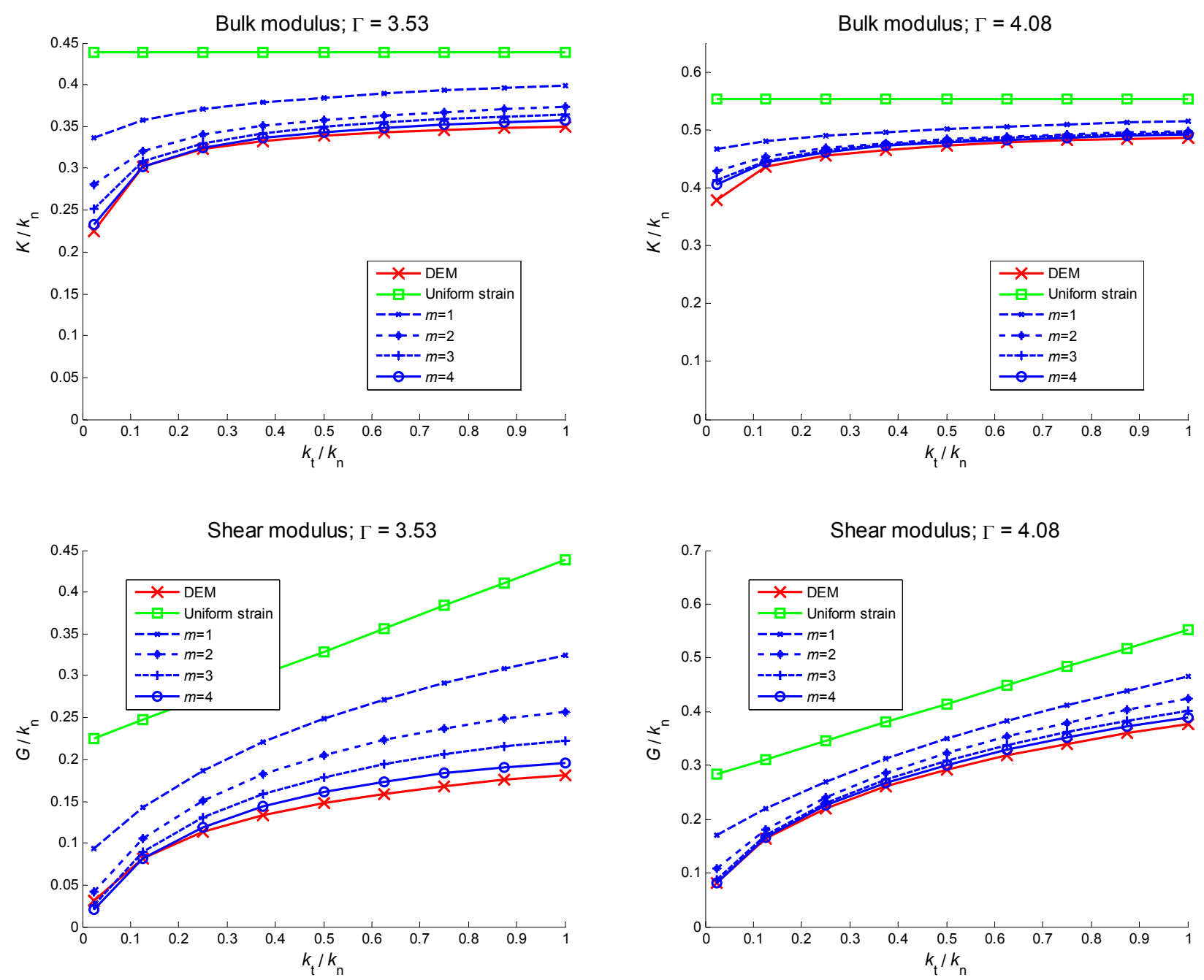

Figure 7. Bulk modulus $K$ and shear modulus $G$ from DEM simulations and according to the proposed theory for two coordination numbers $\Gamma$ and for various stiffness ratios $k_{t} / k_{n}$ and sub-assembly orders $m$. 


$$
\bar{K}_{i j}=\int_{0}^{2 \pi} E(\theta) K_{i j}(\theta) d \theta=\left[\begin{array}{ccccccc}
\frac{k_{n}+k_{t}}{2} & 0 & 0 & -\frac{k_{n}+k_{t}}{2} & 0 & 0 \\
0 & \frac{k_{n}+k_{t}}{2} & 0 & 0 & -\frac{k_{n}+k_{t}}{2} & 0 \\
0 & 0 & \bar{R}^{2} k_{t} & 0 & 0 & \bar{R}^{2} k_{t} \\
-\frac{k_{n}+k_{t}}{2} & 0 & 0 & \frac{k_{n}+k_{t}}{2} & 0 & 0 \\
0 & -\frac{k_{n}+k_{t}}{2} & 0 & 0 & \frac{k_{n}+k_{t}}{2} & 0 \\
0 & 0 & \bar{R}^{2} k_{t} & 0 & 0 & \bar{R}^{2} k_{t}
\end{array}\right]
$$

where the latter identity has been obtained by employing the isotropic contact distribution function $E(\theta)=1 /(2 \pi)$ and by replacing the local particle radii $R^{p}$ and $R^{q}$ in Eq. (14) by the average particle radius $\bar{R}$. Note that the element matrix $\boldsymbol{K}^{p q}$ in Eq. (14) depends only on the contact orientation $\theta^{p q}$

The similar procedure for the right-hand side vector in Eq. (16) gives a vector

$$
\bar{R}_{i}=\int_{0}^{2 \pi} E(\theta) K_{i j}(\theta) U_{j}^{\alpha}(\theta) d \theta=0
$$

where $U^{\alpha, c}$ is the vector of displacements and rotations according to the mean field, as defined in Eq. (17). The right-hand side equals zero in the preceding equation, since the resultant force due to the mean-field deformation is zero on average (but not for individual particles). Thus, it is not meaningful to replace the local contact stiffness matrix in the right-hand side of Eq. (16) by an average.

After replacing the local stiffness matrix by the average in the left-hand side of Eq. (16), the resulting equations become

$$
\left(\sum_{c \in C(p ; m)} \boldsymbol{T}^{c T} \cdot \overline{\boldsymbol{K}} \cdot \boldsymbol{T}^{c}\right) \cdot \boldsymbol{V}^{p}=\sum_{c \in C(p ; m)}\left(\boldsymbol{T}^{c T} \cdot \boldsymbol{K}^{c} \cdot \boldsymbol{U}^{\alpha, c}\right)
$$

By solving these equations for each particle consecutively, a displacement and rotation field $\left\{U_{i}^{*} p, \Omega^{*} p\right.$ is obtained once again, and hence the moduli can be determined.

A comparison of the results employing the local contact stiffness matrices and employing the average contact stiffness matrix is given in Figure 8. The predictions of the shear modulus $G$ employing the average contact stiffness matrix deviate much more from the true DEM-value than 


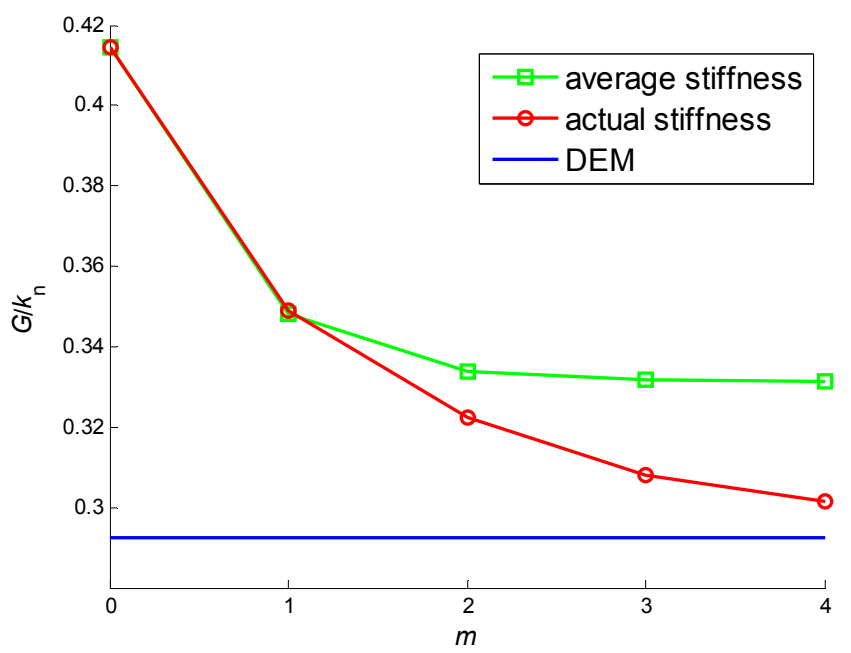

Figure 8. Comparison of the predictions for the shear modulus $G$ using the actual contact stiffness matrix or the average contact stiffness matrix. Results are shown for various sub-assembly orders $m$, where $m=0$ corresponds to the uniform strain result Eq. (21). Case with coordination number $\Gamma=4.08$ and stiffness ratio $k_{t} / k_{n}=0.5$.

the predictions employing the local contact stiffness matrix, except for $m=1$. Furthermore, increasing the sub-assembly order $m$ hardly improves the prediction for $m \geq 2$. Thus, employing an average contact stiffness matrix significantly degrades the quality of the prediction, except for $m=1$.

\section{4. 'Filtering' vs. suppression of near-singular modes}

In Section 4 it has been described how singular and near-singular modes have been suppressed by 'filtering' out some contacts according to the criteria given there. An alternative is to retain all contacts and account for the near-singularity when solving the linear system of equations Eq. (16). This can be done by employing the Moore-Penrose pseudo-inverse (see for example Golub \& van Loan, 1996) for the matrix on the left-hand side of Eq. (16). The results are effectively the same, as the relative differences in predicted moduli between the two approaches are smaller than $0.1 \%$.

\subsection{Moduli in the limit case of zero stiffness ratio}

In many micromechanical studies, assemblies of disks with $k_{t}=0$, i.e. with only central forces, are considered (see for example O' Hern et al., 2003; Majmudar et al., 2007; Ellenbroek et al., 2009). In these studies scaling relations are observed for coordination number, pressure and bulk 
and shear moduli near the 'isostatic' coordination number (“jamming point”). This 'isostatic' limit $\Gamma_{\text {iso }}$, at which the system is statically determinate, corresponds to $\Gamma_{\text {iso }}=4$ in the two-dimensional case considered here when $k_{t}=0$ (Roux, 2000). Note that for cases where $k_{t}>0$, stable packings are possible for $\Gamma \geq 3$ in the two-dimensional case. Expressed in terms of coordination number $\Gamma$, the scaling relations for bulk and shear modulus are

$$
K \propto\left(\Gamma-\Gamma_{\text {iso }}\right)^{r_{K}} \quad G \propto\left(\Gamma-\Gamma_{\text {iso }}\right)^{r_{G}}
$$

where $r_{K}$ and $r_{G}$ are the exponents in the scaling relation for the bulk and shear modulus, respectively. The exponents $r_{K}$ and $r_{G}$ depend on the nature of the particle interaction (O' Hern et al., 2003). Generally, it is found that $r_{G}>r_{K}$. For the two-dimensional case of linear springs, O'Hern et al. (2003) find $r_{K}=0.01$, while Majmudar et al. (2007) give $r_{K}=0.1$. Note that a small positive exponent corresponds to a rapid change in modulus near the isostatic coordination number. Furthermore, Ellenbroek et al. (2009) report different scaling exponents for the bulk modulus, depending on the way the elastic network is generated.

To investigate whether there are significant differences with the case $k_{t}>0$, DEM simulations with various small values of the stiffness ratio $k_{t} / k_{n}$, at constant coordination number, have been performed of hydrostatic compression. In hydrostatic compression, tangential forces are zero on average. One would then expect that the tangential stiffness $k_{t}$ has only a minor effect on the bulk modulus $K$. In fact, according to the uniform strain assumption Eq. (21), the tangential stiffness $k_{t}$ has no effect on the bulk modulus $K$.

Results for the bulk moduli $K$ from the DEM simulations are shown in Figure 9. For $\Gamma=3.53$, below the 'isostatic' limit, the value of the bulk modulus for $k_{t} / k_{n} \rightarrow 0$ is zero and the bulk modulus scales linearly with stiffness ratio. For $\Gamma=4.08$, with coordination number just above the isostatic limit, the limit value of the bulk modulus, $K\left(k_{t} / k_{n} \rightarrow 0\right)$, is non-zero, although a rapid change in bulk modulus is observed for small stiffness ratios: the ratio $K\left(k_{t} / k_{n}=1\right) / K\left(k_{t} / k_{n} \rightarrow 0\right) \cong 40$. For a denser assembly with coordination number $\Gamma=4.29$ (further away from the isostatic limit; this case has not been considered before), the ratio $K\left(k_{t} / k_{n}=1\right) / K\left(k_{t} / k_{n} \rightarrow 0\right) \cong 2.5$. For completeness, qualitatively similar results for the shear modulus $G$ are also shown in Figure 9.

These results show that small variations in tangential stiffness $k_{t}$ have a large quantitative effect on macroscopic properties, even on the bulk modulus $K$, for which it could be expected that it does not depend on $k_{t}$. This sensitivity is especially pronounced for systems with coordination numbers below, or near, the isostatic limit. Thus, caution should be exercised when interpreting results from DEM simulations or theories that employ only central forces $\left(k_{t}=0\right)$. 

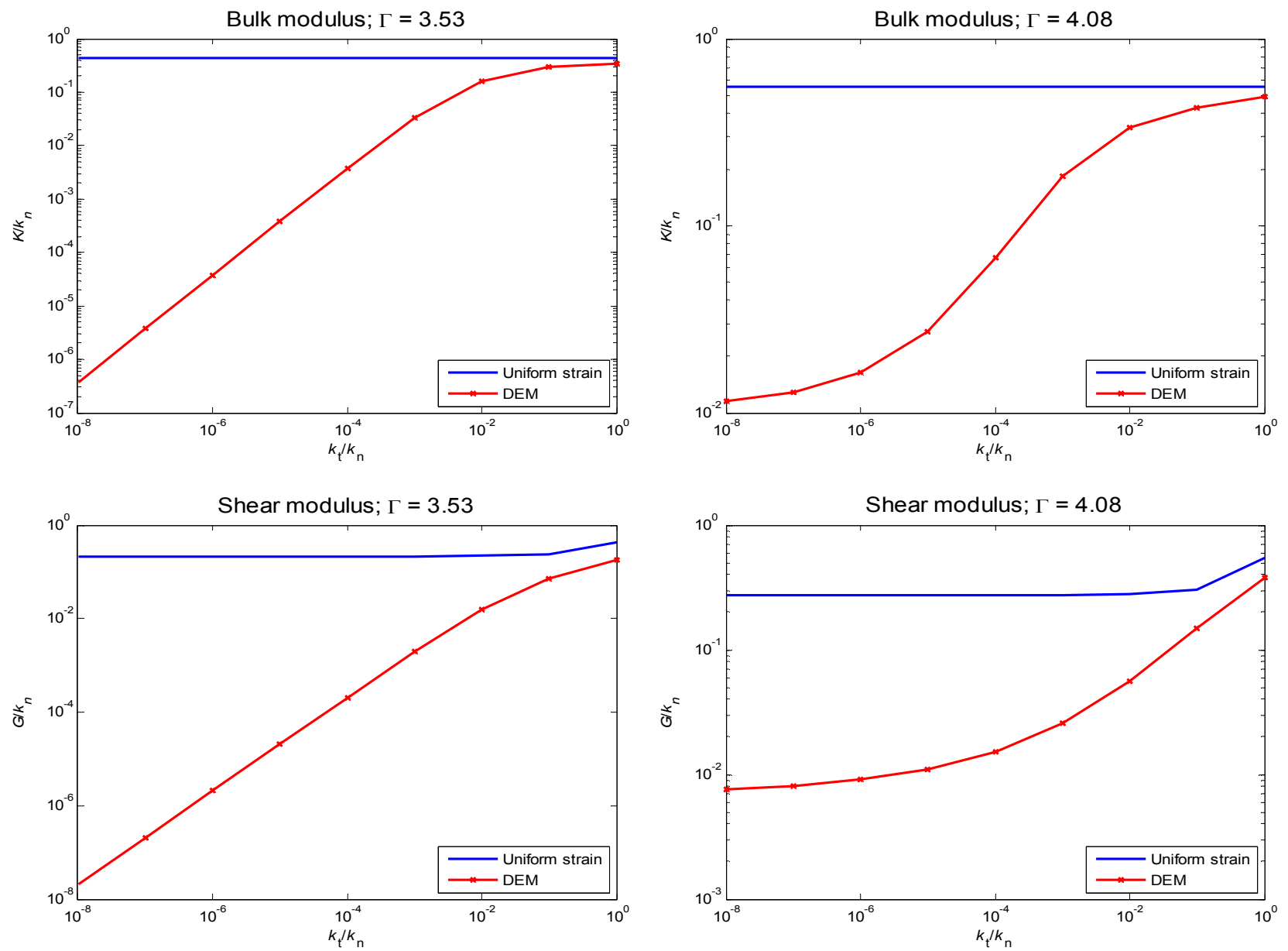

Figure 9. Log-log plots of bulk modulus $K$ (top row) and shear modulus $G$ (bottom row) vs. stiffness ratio $k_{t} / k_{n}$ for $\Gamma=3.53$ (left column) and for $\Gamma=4.08$ (right column). Results are from DEM simulations. Also shown is the prediction Eq. (21) according to the uniform strain assumption. For $\Gamma=3.53, K^{\prime} k_{n}$ and $G^{\prime} k_{n}$ scale as $k_{t} / k_{n}$ for small stiffness ratios.

It has been suggested in another study (Wyart, 2005) that the ratio $G^{\prime} K$ of shear modulus over bulk modulus scales linearly with the 'distance' from the isostatic coordination number $\Gamma_{\text {iso }}$ in the case with $k_{t}=0$

$$
\frac{G(\Gamma)}{K(\Gamma)} \propto \Gamma-\Gamma_{\text {iso }}
$$

Note that the ratio $G^{\prime} / K$ is related to the macroscopic Poisson ratio, see Eq. (20).

The scaling relation Eq. (26) has been confirmed by computer simulations for frictionless assemblies with $k_{t}=0$ (O'Hern et al., 2003), as well as for frictional assemblies (Somfai et al., 2007). Since the stiffness ratio $k_{t} / k_{n}$ represents another measure of 'distance' from an isostatic state, it is interesting to investigate the influence of the stiffness ratio on the ratio of the moduli for small stiff- 


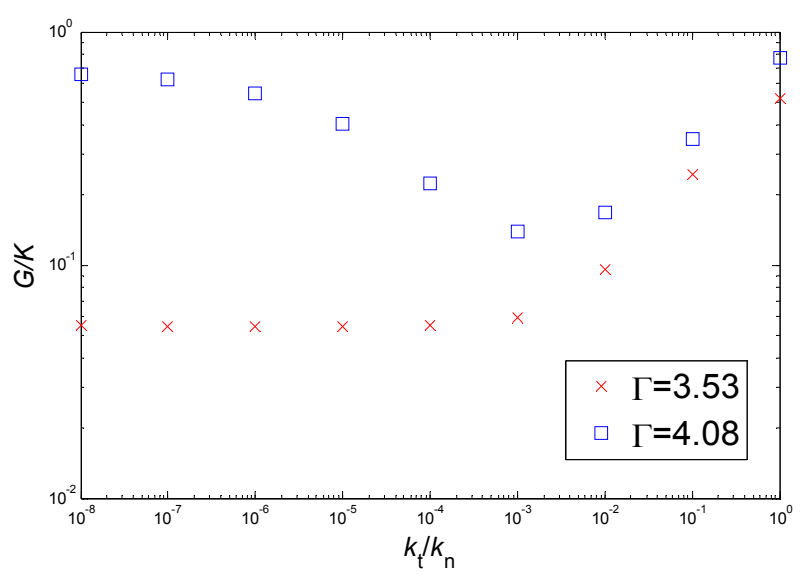

Figure 10. Ratio of elastic moduli $G^{\prime} K$ as a function of stiffness ratio $k_{t} / k_{n}$ for two values of coordination number $\Gamma$.

ness ratios. This is done for the two values of coordination number $\Gamma=3.53$ and $\Gamma=4.08$. The results from the DEM simulations are shown in Figure 10. For small stiffness ratio $k_{t} / k_{n}$, $G\left(k_{t} / k_{n}\right) / K\left(k_{t} / k_{n}\right)$ becomes constant.

\section{Discussion}

A method has been developed for obtaining micromechanical predictions of the macroscopic elastic moduli of loose two-dimensional isotropic assemblies. The method is based on predictions of the particle displacement and rotation fields that are determined from the equilibrium equations for small sub-assemblies of various sizes. From these fields, predictions of the moduli can be obtained from either a stress-based or an energy-based approach. It is found that generally the stress-based approach gives a more accurate prediction. It remains an open question why this is the case.

By increasing the order (or size) $m$ of the sub-assemblies, the predictions get closer to the true moduli determined from DEM simulations. The present predictions are much closer to the true moduli than those according to the uniform strain assumption, which can be off by a factor $2-3$ for the loosest assembly with coordination number $\Gamma=3.53$. The improvement of the prediction for subassembly order $m \geq 2$ is especially pronounced for the shear modulus of the loosest assembly with $\Gamma=3.53$. Quantitatively, the shear modulus is already fairly well predicted for $m=2$ with average relative deviations of $38 \%$ and $13 \%$ for $\Gamma=3.53$ and $\Gamma=4.08$, respectively. For $m=3$, these average relative deviations are $16 \%$ and $6 \%$, while for $m=4$, these deviations are $2 \%$ and $2 \%$. 


\section{N.P. Kruyt \& I. Agnolin \& S. Luding \& L. Rothenburg}

These results give indications of the size of sub-assembly $m$ that has to be considered, for a required level of accuracy. In analytical formulations, additional assumptions are required for geometrical averages, like effective average particle stiffnesses. By employing a semi-numerical approach here, this source of errors is excluded here.

As a step towards an analytical formulation of the present approach, it has been investigated whether it is possible to replace the sum of the local contact stiffness matrices by a suitable average contact stiffness matrix in the solution of the equilibrium conditions for the sub-assemblies. It is found that this leads to a clear deterioration of the predictions, except for the smallest sub-assembly order $m=1$. This deterioration may prove to be an impediment to accurate analytical formulations of the current approach.

It would be expected that the tangential stiffness $k_{t}$ has only a small influence on the macroscopic bulk modulus $K$. Results from DEM simulations of hydrostatic compression of loose systems, below and slightly above the isostatic limit, and with various small values of the stiffness ratio $k_{t} / k_{n}$ show that $K$ strongly depends on $k_{t} / k_{n}$ near $k_{t} / k_{n}=0$. Thus, caution should be exercised when interpreting results from DEM simulations or theories that employ only central forces $\left(k_{t}=0\right)$. It is observed from the DEM simulations that the ratio of the elastic moduli $G^{\prime} K$ is constant for small stiffness ratios.

In compressive loading, tangential forces at contacts are zero on average. Therefore the role of particle rotations is small. In shearing loading, tangential forces at contacts are not zero on average. An accurate prediction of the particle rotations is difficult, as they are rather sensitive to the contact geometry (Agnolin \& Kruyt, 2008). This forms one reason why it is more difficult to accurately predict the shear modulus than the bulk modulus.

A different method for predicting the elastic moduli is the "pair-fluctuation" approach (Jenkins et al., 2005; Agnolin et al., 2006; La Ragione \& Jenkins, 2007). There, equilibrium equations are formulated for a pair of particles that correspond to a single contact. From the approximated equilibrium equations, the relative displacement at contacts $\Delta_{i}^{c}$ is obtained analytically in terms of geometrical characteristics of the assembly. The two main differences with the current approach are that: (i) additional assumptions have to be made to facilitate analytical derivations and (ii) the resulting relative displacements $\left\{\Delta_{i}^{c}\right\}$ are not necessarily compatible. The relative displacements are compatible if displacement and rotation fields $\left\{U_{i}^{p}, \Omega^{p}\right\}$ exist such that the relative displacements $\left\{\Delta_{i}^{c}\right\}$ are given by Eq. (3).

In the present approach complete knowledge of the contact geometry is required. This information is generally only available from DEM simulations. It is an open question what statistical measures are sufficient for the description of the contact geometry. For the isotropic systems considered 
here the primary statistical measure is coordination number. Two extreme cases are: (i) dense systems with coordination number around 6 and (ii) loose systems near the isostatic limit. For dense systems coordination number is considered a sufficient measure of the contact geometry. For loose systems just above the isostatic limit no statistical measure may be sufficient: the full knowledge of the contact geometry is required, since a small change in contact geometry leads to large rearrangement of contact forces. The theoretical "pair-fluctuation" approach (as referred to above) involves, as a statistical measure of the contact geometry, the conditional contact distribution function, which gives the probability of finding a contact at an orientation given that another contact with certain orientation is present. The theory of La Ragione \& Jenkins (2007) additionally involves the standard deviation in particle coordination number as a statistical measure of the contact geometry. This standard deviation is directly connected to the coordination number, as was shown by Magnanimo et al. (2008), using DEM simulations.

The current approach is based on the solution of particle displacements and rotations from the force and moment equilibrium equations. A dual approach consists of the solution of contact forces from compatibility equations for relative displacements (Kruyt \& Rothenburg, 1996; Kruyt, 2003). This approach has been pursued analytically by Trentadue $(2001,2004)$ and semi-numerically by Kruyt \& Rothenburg (2002) for a sub-assembly order $m=1$ for the case without particle rotations. Extension to the cases with particle rotations and with larger sub-assembly orders $m$ will be pursued, as this will yield rigorous lower bounds for the moduli (besides upper bounds based on the energy-based approach, see Section 5.1), based on the minimum complementary energy principle.

It is interesting to determine correlation functions for the fluctuations in particle displacements and rotations from the DEM simulations. Maloney (2006) observed that the correlation function scales with the system size in his study of the elastic response of dense random packings of disks with central interaction, i.e. with $k_{t}=0$. Thus, in that study particle rotations are all zero, contrary to the case studied here.

Future work will deal with the extension of the present approach to the study of different particle shapes and of anisotropic and three-dimensional systems, as well as with analytical formulations of the current approach.

\section{Acknowledgment}

The reviewers are thanked for their valuable comments and suggestions that have helped to improve the manuscript. 


\section{References}

Agnolin, I. \& Jenkins, J.T. \& La Ragione, L. (2006). A continuum theory for a random array of identical, elastic, frictional disks. Mechanics of Materials 38 687-701.

Agnolin, I. \& Kruyt, N.P. (2008). On the elastic moduli of two-dimensional assemblies of disks: relevance and modelling of fluctuations in particle displacements and rotations. Computers and Mathematics with Applications 55 245-256.

Agnolin, I. \& Roux, J.N. (2007a). Internal states of model isotropic granular packings. I. Assembling process, geometry, and contact networks. Physical Review E 76061302.

Agnolin, I. \& Roux, J.N. (2007b). Internal states of model isotropic granular packings. III. Elastic properties. Physical Review E 76061304.

Agnolin, I. \& Roux, J.N. (2008). On the elastic moduli of three-dimensional assemblies of spheres: characterization and modelling of fluctuations in the particle displacement and rotation. International Journal of Solids and Structures 45 1101-1123.

Bathurst, R.J. \& Rothenburg, L. (1988a). Micromechanical aspects of isotropic granular assemblies with linear contact interactions. Journal of Applied Mechanics (Transactions of the ASME) 55 1723.

Bathurst, R.J. \& Rothenburg, L. (1988b). Note on a random isotropic granular material with negative Poisson's ratio. International Journal of Engineering Science 26 373-383.

Calvetti, F \& Tamagnini, C. \& Viggiani, G. (2002). On the incremental behaviour of granular soils, Proceedings NUMOG VIII, pp.3-9, eds G. Pande and S. Pietruszczak, Swets and Zeitlinger, Lisse, The Netherlands.

Cambou, B. \& Dubujet, P. \& Emeriault, F. \& Sidoroff, F. (1995). Homogenisation for granular materials. European Journal of Mechanics A / Solids 14 255-276.

Chang, C.S. \& Liao, C.L. (1994). Estimates of elastic modulus for media of randomly packed granules. Applied Mechanics Review 47 S197-S206.

Chang, C.S. \& Misra, A. \& Sundaram, S.S. (1990). Micromechanical modelling of cemented sands under low amplitude oscillations. Géotechnique 40 251-263.

Christoffersen, J. \& Mehrabadi, M.M. \& Nemat-Nasser, S. (1981). A micromechanical description of granular material behaviour. Journal of Applied Mechanics (Transactions of the ASME) $\mathbf{4 8}$ 339-344.

Cundall, P.A. \& Strack, O.D.L. (1979). A discrete numerical model for granular assemblies. Géotechnique 9 47-65. 
Digby, P.J. (1981). The effective moduli of porous granular rock. Journal of Applied Mechanics (Transactions of the ASME) 48 803-808.

Drescher, A. \& de Josselin de Jong, G. (1972). Photoelastic verification of a mechanical model for the flow of a granular materials. Journal of the Mechanics and Physics of Solids 20 337-351.

Ellenbroek, W.G. \& Zeravcic, Z. \& van Saarloos, W. \& van Hecke, M. (2009). Non-affine response: jammed packings versus spring networks. EPL 8734004.

Eringen, A.C. (1999). Microcontinuum field theories: foundations and solids. Springer-Verlag, New York, USA.

Goldhirsch, I. \& Goldenberg, C. (2002). On the microscopic foundations of elasticity. European Physical Journal E 9 245-251.

Goldenberg, C. \& Goldhirsch, I. (2002). Force chains, microelasticity, and macroelasticity. Physical Review Letters 89084302.

Golub, G.H. \& van Loan, C.F. (1996). Matrix computations. The Johns Hopkins University Press, Baltimore, USA.

Hill, R. (1950). The mathematical theory of plasticity. Clarendon Press, Oxford, UK.

Hoque, E. \& Tatsuoka, F. (2004). Effects of stress ratio on small-strain stiffness during triaxial shearing. Géotechnique 54, 429-439.

Horne, M.R. (1965). The behaviour of an assembly of rotound, rigid, cohesionless particles I and II, Proceedings of the Royal Society of London A 286 62-97.

Jenkins, J.T. \& Johnson, D \& La Ragione, L \& Makse, H.A. (2005). Fluctuations and the effective moduli of an isotropic, random aggregate of identical, frictionless spheres. Journal of the Mechanics and Physics of Solids 53 197-225.

Johnson, K.L. (1985). Contact mechanics. Cambridge University Press, Cambridge, UK.

Kruyt, N.P. (2003). Statics and kinematics of discrete Cosserat-type granular materials. International Journal of Solids and Structures 40 511-534.

Kruyt, N.P. \& Rothenburg, L. (1996). Micromechanical definition of the strain tensor for granular materials. Journal of Applied Mechanics (Transactions of the ASME) 63 706-711.

Kruyt, N.P. \& Rothenburg, L. (1998). Statistical theories for the elastic moduli of two-dimensional assemblies of granular materials. International Journal of Engineering Science 36 1127-1142.

Kruyt, N.P. \& Rothenburg, L. (2002). Micromechanical bounds for the elastic moduli of granular materials. International Journal of Solids and Structures 39 311-324.

Kruyt, N.P. \& Rothenburg, L. (2004). Kinematic and static assumptions for homogenization in micromechanics of granular materials. Mechanics of Materials 36 1157-1173. 
Kuhn, M.R. \& Bagi, K. (2009). Specimen size effect in discrete element simulations of granular assemblies. Journal of Engineering Mechanics 135 485-492.

La Ragione, L. \& Jenkins, J.T. (2007). The initial response of an idealized granular material. Proceedings of the Royal Society of London A 463 735-758.

Luding, S. (2004). Micro-macro transition for anisotropic, frictional granular packings. International Journal of Solids and Structures 41 5821-5836.

Magnanimo, V. \& La Ragione, L. \& Jenkins, J.T. \& Wang, P. \& Makse, H. A. (2008). Characterizing the shear and bulk moduli of an idealized granular material. EPL 8134006.

Majmudar, T.S. \& Sperl, M. \& Luding, S. \& Behringer, R.P. (2007). Jamming transition in granular systems. Physical Review Letters 98058001.

Maloney, C.E. (2006). Correlations in the elastic response of dense random packings. Physical Review Letters 97035503.

Makse, H.A. \& Gland, N. \& Johnson, D.L. \& Schwartz, L.M. (1999). Why effective medium theory fails in granular materials. Physical Review Letters 83 5070-5073.

O’ Hern, C. S. \& Silbert, L. E. \& Liu, A. J. \& Nagel, S.R. (2003). Jamming at zero temperature and zero applied stress: the epitome of disorder. Physical Review E $\mathbf{6 8} 011306$.

Quickenden, T.I. \& Tan, G.K. (1974). Random packing in two dimensions and the structure of monolayers, Journal of Colloid Interface Science 48 382-393.

Rothenburg, L. (1980). Micromechanics of idealised granular materials. PhD Thesis Department of Civil Engineering, Carleton University, Ottawa, Ontario, Canada.

Rothenburg, L. \& Bathurst, R.J. (1992). Micromechanical features of granular assemblies with planar particles. Géotechnique 42 79-95.

Rothenburg, L. \& Berlin, A.A. \& Bathurst, R.J. (1991). Microstructure of isotropic materials with negative Poisson's ratio. Nature 354 470-472.

Rothenburg, L. \& Kruyt, N.P. (2001). On limitations of the uniform strain assumption in micromechanics of granular materials. Powders \& Grains 2001: $4^{\text {th }}$ International Conference on Micromechanics of Granular Media, pp.191-194, ed. Y. Kishino, Balkema Publishers, Lisse, The Netherlands.

Rothenburg, L.\& Kruyt, N.P. (2004). Critical state and evolution of coordination number in simulated granular materials. International Journal of Solids and Structures 41 5763-5774.

Rothenburg, L.\& Kruyt, N.P. (2009). Micromechanical definition of an entropy for quasi-static deformation of granular materials. Journal of the Mechanics and Physics of Solids 57 634-655.

Roux, J.N. (2000). Geometric origin of mechanical properties of granular materials. Physical Review E $616802-6836$. 
Somfai, E. \& Roux, J.N. \& Snoeijer, J.H. \& van Hecke, M. \& van Saarloos, W. (2005). Elastic wave propagation in confined granular system. Physical Review E 72021301.

Somfai, E. \& van Hecke, M. \& Ellenbroek, W.G. \& Shundyak, K. \& van Saarloos, W. (2007). Critical and noncritical jamming of frictional grains. Physical Review E $75020301 \mathrm{R}$.

Tatsuoka, F. (1999). Small strain behaviour of granular materials. In: Mechanics of Granular Materials: an introduction, pp.299-308, eds. M. Oda \& K. Iwashita, Balkema Publishers, Rotterdam, The Netherlands.

Trentadue, F. (2001). A micromechanical model for a non-linear elastic granular material based on local equilibrium conditions. International Journal of Solids and Structures 38 7319-7342.

Trentadue, F. (2004). An equilibrium-based approach for the micromechanical modelling of a nonlinear elastic granular material. Mechanics of Materials 36 323-334.

Walton, K. (1987). The effective elastic moduli of a random packing of spheres. Journal of the Mechanics and Physics of Solids 35 213-226.

Washizu, K. (1968). Variational methods in elasticity and plasticity. Pergamon Press, Oxford, UK.

Wyart, M. (2005). On the rigidity of amorphous solids. Annales de Physique 30 1-96.

Zienkiewicz, O.C. \& Taylor, R.L. (1987). The finite element method: basic formulation and linear problems. McGraw-Hill, London, UK. 